\title{
Effects of plasma current on nonlinear interactions of ITG turbulence, zonal flows and geodesic acoustic modes
}

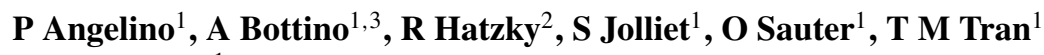 \\ and $L$ Villard ${ }^{1}$ \\ ${ }^{1}$ Ecole Polytechnique Fédérale de Lausanne (EPFL), Centre de Recherches en Physique des \\ Plasmas, Association Euratom Confédération Suisse, CH-1015, Lausanne, Switzerland \\ ${ }^{2}$ Computer Center of the Max-Planck-Gesellschaft and the Institute for Plasma Physics, D-85748 \\ Garching, Germany
}

Received 24 November 2005

Published 15 March 2006

Online at stacks.iop.org/PPCF/48/557

\begin{abstract}
The mutual interactions of ion temperature gradient (ITG) driven modes, zonal flows and geodesic acoustic modes (GAM) in tokamak plasmas are investigated using a global nonlinear gyrokinetic formulation with totally unconstrained evolution of temperature gradient and profile. A series of numerical simulations with the same initial temperature and density profile specifications is performed using a sequence of ideal MHD equilibria differing only in the value of the total plasma current, in particular with identical magnetic shear profiles and shapes of magnetic surfaces. On top of a bursty or quasi-steady state behaviour the zonal flows oscillate at the GAM frequency. The amplitude of these oscillations increases with the value of the safety factor $q$, resulting in a less effective suppression of ITG turbulence by zonal flows at a lower plasma current. The turbulence-driven volume-averaged radial heat transport is found to scale inversely with the total plasma current.
\end{abstract}

(Some figures in this article are in colour only in the electronic version)

\section{Introduction}

In virtually all tokamak experiments, the particle and heat losses largely exceed the theoretical predictions based on the neoclassical theory. In the neoclassical model, binary collisions are the basic mechanism by which energy and particles are transported across the magnetic field lines. Although the reason for the difference between the measured radial transport and the neoclassical prediction is not yet completely understood, the main source of anomalous

${ }^{3}$ Present address: Max-Planck Institut für Plasmaphysik, IPP-EURATOM Association, Garching, Germany. 
transport is now mainly attributed to plasma turbulence [1] driven by free energy sources in the plasma. These small-scale plasma fluctuations arise from instabilities driven by temperature and density gradients always present in any magnetic confinement device. These instabilities are often called microinstabilities because they are characterized by small spatial scales as compared with the size of the tokamak (but large compared with the Debye length above which the plasma is quasi-neutral) and by low frequencies as compared with the plasma frequency, $\omega_{\text {pe }}$. The physics governing plasma turbulence is extremely complex because of the wide range of spatial and time scales involved in the collective turbulent motion of plasma particles. Therefore, a comprehensive theoretical model for anomalous transport does not yet exist which can explain the high heat conductivity found in the experiments. Most existing transport models are based on a mixing length rule based on quasi-linear stability theory. The fact that the nonlinear processes rather than linear quantities determine the scaling of the ion heat conductivity places considerable limitations on the applicability of these models. The ion heat anomalous transport in the plasma core seems to be dominated by a class of microinstabilites, the toroidal ion temperature gradient (ITG) driven modes [2]. ITG turbulence is known to self organize to form coherent macroscopic structures [3]. These structures take the form of a macroscopic electrostatic potential which depends only on the radial coordinate. The $E \times B$ poloidal flows associated with this potential are referred to as zonal flows. Both experimental [4] and theoretical evidence [5] have been supplied which demonstrate that zonal flows play a crucial role in regulating the nonlinear evolution of ITG turbulence. A comprehensive review of the physics of zonal flows is given in [6]. This effect relies on the capability of the zonal flows to shear the radial coherent turbulence structure. Introducing the flux surface average of the electrostatic potential $\bar{\phi}$, the radial and toroidal correlation lengths of turbulence, $\Delta \psi$ and $\Delta \varphi$ respectively, the shearing rate

$$
\omega_{E \times B}=\frac{\Delta \psi}{\Delta \varphi} \frac{\mathrm{d}^{2}}{\mathrm{~d} \psi^{2}} \bar{\phi}(\psi)
$$

is a measure of the shearing action of the zonal flows on the global mode structure. When $\omega_{E \times B}$ becomes comparable to the decorrelation rate of the ambient turbulence, the latter is strongly suppressed.

In toroidal systems such as tokamaks, zonal flows can oscillate in time by coupling with poloidally asymmetric $(m, n)=(1,0)$ pressure perturbations [7]. These oscillations are called the geodesic acoustic modes (GAM) since the coupling comes through the geodesic curvature. The importance of GAM oscillations resides in the different shearing efficiency zonal flows have in relation to their oscillatory behaviour. While the stationary zonal flows suppress turbulence efficiently, fluctuations make the suppression of the turbulence by the zonal flows less effective. In order to take into account this effect, an effective shearing rate $\omega_{\text {Eff }} \lesssim \omega_{E \times B}$ has been introduced [8]. In a study of tokamak edge turbulence [9], it was shown that geodesic curvature inhibits the turbulence suppression by limiting the growth of steady zonal flows. Recently, it has been pointed out [10] that the stationary or oscillatory nature of zonal flow behaviour is critically dependent on the value of the safety factor $q$. All these arguments suggest that a series of relationships can be established between turbulence, zonal flow, GAM and $q$, which in turn depends on the plasma current profile. To investigate these interactions we have run a series of numerical simulations with the global nonlinear gyrokinetic code ORB5 [11]. The results are illustrated in the present work.

This paper is organized as follows. The model and its implementation in the numerical code ORB5 are described in section 2. The magnetic equilibrium and the parameters of the simulations are presented in section 3 . The heat flux and ion conductivity are analysed in section 4 . Section 5 gives a detailed description of the heat flux modulation by the zonal flows. 
The effects of GAM oscillations on turbulent transport are investigated in section 6 . The results are summarized in section 7 .

\section{The model}

The simulations are based on the electrostatic gyrokinetic equations derived by Hahm [12]. In the code ORB5 [13], ion motion is described by means of the nonlinear gyrokinetic Vlasov equation. An adiabatic model is used for electrons, where the flux surface averaged part of the potential has been subtracted in order to obtain the correct zonal flow amplitude [14]. The system is closed by the Poisson equation that, in the long wavelength approximation, reduces to quasi-neutrality equation. Finally, the polarization density is linearized with the ansatz: $n_{\mathrm{i}} \simeq n_{0}+\delta n$. This set of equations has been proved to be energy and particle number conserving [15]. A finite element scheme (FEM) is used for the Poisson solver. The fivedimensional particle phase space is sampled with a particle in cell (PIC) scheme, and a control variate $(\delta f)$ method is used in order to reduce noise [16]. Time integration is provided by a 4th order Runge-Kutta scheme. A canonical Maxwellian is used as an equilibrium distribution function in order to avoid spurious drive of zonal flows [17]. No approximations (besides axisymmetry) were made on the geometry of the magnetic field. ORB5 is interfaced with the code CHEASE [18], which supplies ideal MHD equilibria or experimental magnetic configuration reconstruction. In our simulations, neither the temperature, nor its gradient, nor the heat flux is constrained. The temperature and its gradient evolve consistently with each other. The MHD equilibrium is not evolved in time consistently with the evolution of the temperature profile of the gyrokinetic simulation. The reason is that the electrostatic approximation implies considering very low $\beta$ plasmas. For these equilibria the geometry of the magnetic surfaces is rather insensitive to the variation of the pressure profile. The code ORB5 has been qualitatively benchmarked against the global PIC code GT3D [17] and on a linear CYCLONE case [19]. A zonal flow damping [20] test has been successfully performed.

\section{Simulation parameters}

The analysis presented in this paper is based on a series of five simulations where the total plasma current has been varied in five steps: 1, 1.33, 1.66, 2 and 2.33 times a basic value of $I_{\mathrm{N} 0}=0.258$, where $I_{\mathrm{N}}$ is the normalized current $I_{\mathrm{N}}=I_{\mathrm{P}} / a B$. Here, $I_{\mathrm{P}}$ is the plasma current [MA], $a$ the minor radius on equatorial plane [m] and $B$ the vacuum magnetic field at the centre of the discharge [T]. We vary the total plasma current by rescaling the current density profile, which is equivalent to rescaling the safety factor $\left(q_{\mathrm{s}}\right)$ profile of figure 1 . The other parameters are kept constant (in particular the shape of the magnetic surfaces of figure 2 is unchanged). We label these simulations as $1 \mathrm{x}, 1.3 \mathrm{x}, 1.6 \mathrm{x}, 2 \mathrm{x}$ and $2.3 \mathrm{x}$ according to the total current value. The sequence of equilibria is carefully chosen in such a way as to isolate the effect of plasma current ( $q_{\mathrm{s}}$ value) without changing the magnetic shear, which is known to have important effects on the underlying microinstabilities. Changing both the $q_{\mathrm{s}}$ value and the shear would have made it difficult to disentangle both effects. The basic magnetic configuration is the one reconstructed from a shot of the tokamak TCV. It is an elongated D-shaped plasma (figure 2), with inverse aspect ratio $\epsilon \equiv a / R=0.27$, elongation $\kappa=1.5$ and $a=0.24 \mathrm{~m}=40 \rho_{\mathrm{i}}$. The motivations at the basis of this choice are explained in [21], where a linear stability analysis of this case has been performed. The value of the magnetic field on axis is $B_{0}=1.44 \mathrm{~T}$. Starting from this configuration, some parameters have been retuned to destabilize ion temperature gradient (ITG) modes, since global linear simulations 

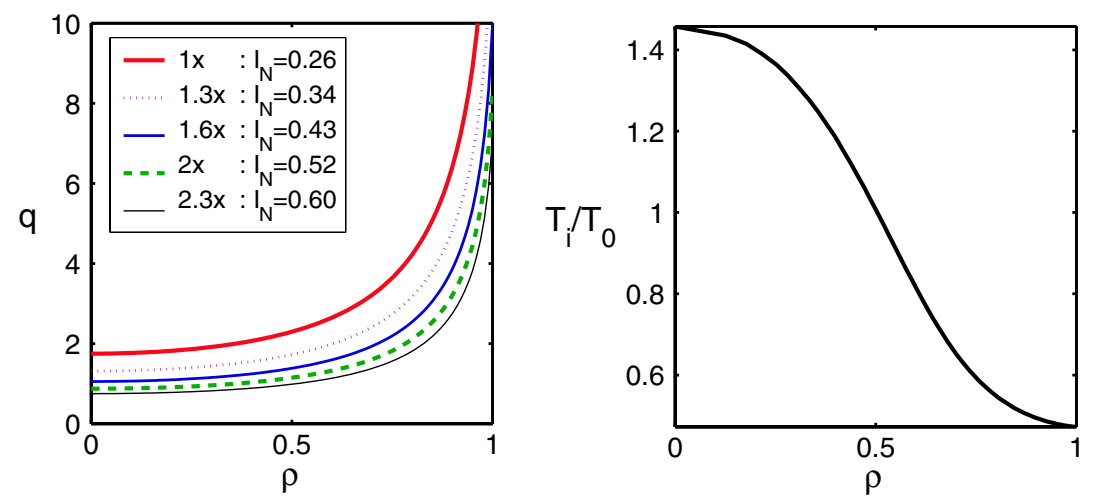

Figure 1. Safety factor $q$ profile and effective ion temperature $T_{\mathrm{i}}$ profile. Ion temperature is normalized to the value $T_{\mathrm{e} 0}$ at $\rho=0.5$.

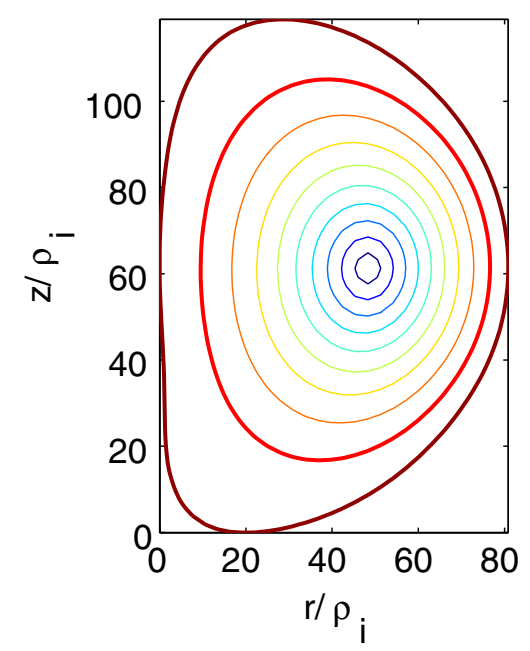

Figure 2. Magnetic equilibrium: poloidal section showing the magnetic surfaces and $r$ and $z$ are the polar coordinates in units of the ion gyroradius $\rho_{\mathrm{i}}$.

with experimental temperature and density profile showed that the ITG mode is marginally stable in this shot [22]. The ITG has been increased, and the effective profile obtained is shown in figure 1. Electron temperature profile is flat, with $T_{\mathrm{e}}=T_{\mathrm{i}}\left(\rho_{0}=0.5\right)$, and the effective initial density $n_{\mathrm{i} 0}=n_{\mathrm{e} 0}$ is almost flat ${ }^{1}$. The equilibrium is low $\beta(<1.2 \%)$ and we therefore neglect finite $\beta$ effects such as the diamagnetic drift of particles. The numerical simulations have been performed with 33 million tracers. The electrostatic potential is solved on a grid $64 \times 128 \times 64$, with toroidal coordinates $\rho=\sqrt{\psi / \psi_{a}}, \theta$ (poloidal angle) and $\varphi$ (toroidal angle). In order to reduce numerical noise, a Fourier filter is applied in both poloidal and toroidal directions. With a linearly most unstable toroidal mode in the interval $4 \leqslant n \leqslant 9$,

1 We talk here of 'effective' profile. Since the initial distribution function is a canonical Maxwellian, the input analytic profile is given as a function of the toroidal canonical momentum $\psi_{0}$. It obviously differs from a radial profile given as a function of $\rho=\sqrt{\psi / \psi_{a}}$, where $\psi$ is the poloidal magnetic flux and $\psi_{a}$ is its value at the plasma boundary. Thus, 'effective' density and temperature profiles are reconstructed from the distribution function and the velocities of the particles at time $t=0$. 


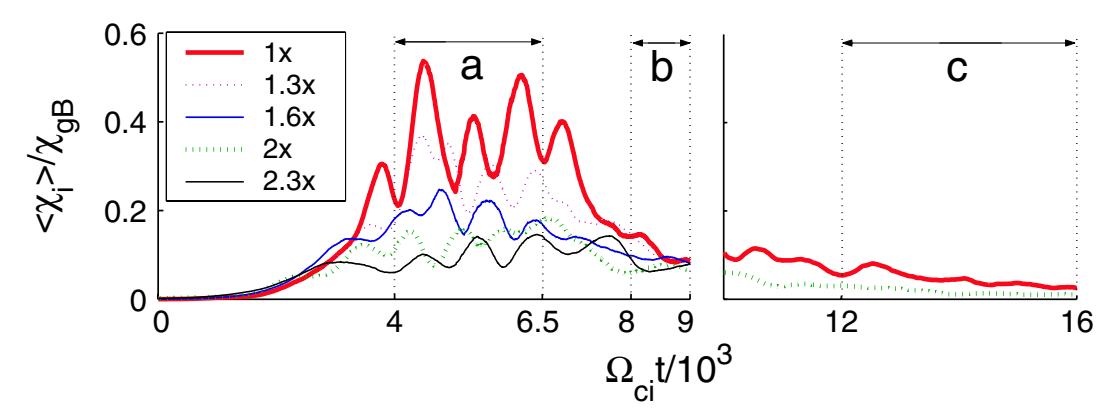

Figure 3. Radial average heat flux and time history for the different current values. The vertical lines indicate the intervals chosen for the analysis: $(a)$ in the burst regime, $(b)$ in the transition phase and $(c)$ in the quasi-steady regime.

and $0.98 \leqslant q\left(\rho_{0}\right) \leqslant 2.30$, a filter window of $0 \leqslant n \leqslant 15$ and $-40 \leqslant m \leqslant 40$ ( $n, m$ are, respectively, the poloidal and toroidal wave numbers) provides the required convergence for all the five current cases. After a convergence test, a time step of $\Delta t \Omega_{\mathrm{ci}}=5$ (where $\Omega_{\mathrm{ci}}$ is the ion cyclotron frequency) has been chosen. The ions considered are hydrogen.

\section{Ion heat transport analysis}

A global quantity, very useful in characterizing our simulations, is the volume averaged radial heat flux $Q$ defined as [23]

$$
Q(\psi)=\frac{1}{\Delta V} \int_{\psi}^{\psi+\Delta \psi} \frac{1}{2} m_{\mathrm{i}} v_{\mathrm{thi}}^{2} \frac{\langle\mathbf{E}\rangle \times \mathbf{h}}{B} \cdot \frac{\nabla \rho}{|\nabla \rho|} \delta f \mathrm{~d} \mathbf{v} \mathrm{d} \mathbf{R},
$$

where $\Delta V$ is the volume of the plasma between the magnetic surfaces $\psi$ and $\psi+\Delta \psi, m_{\mathrm{i}}$ and $v_{\text {thi }}$ are the ion mass and thermal velocity, $\langle\mathbf{E}\rangle$ is the gyroaveraged electric field and $\mathbf{h}=\mathbf{B} / B$. The ion diffusivity $\chi_{\mathrm{i}}$ is defined as

$$
\chi_{\mathrm{i}} \equiv-\frac{Q}{n_{\mathrm{i}} \nabla T_{\mathrm{i}}}
$$

We stress that, unlike usual transport codes which calculate $T$ profiles and heat flux $Q$ using an assumed form for $\chi_{\mathrm{i}}$, here $T$ and $Q$ profiles are obtained from ab initio numerical simulations and the quantity $\chi_{i}$ is constructed from the simulation results. Therefore in this work, no assumption on the scaling of $\chi_{\mathrm{i}}$ is made. Heat flux, ITG $\left(\nabla T_{\mathrm{i}}\right)$ and density $\left(n_{\mathrm{i}}\right)$ profiles are evaluated from the particle velocity and distribution function at each time step. Thus, from equation (3) we obtain a radial profile of $\chi_{i}$ evolving in time. In the following, we will refer to its value normalized to the gyro-Bohm transport coefficient [24]:

$$
\chi_{\mathrm{gB}}=\frac{\rho_{\mathrm{s}}^{2} c_{\mathrm{s}}}{\langle a\rangle} \sim \frac{\rho_{\mathrm{s}}^{2} c_{\mathrm{s}}}{a \sqrt{\kappa}},
$$

where $c_{\mathrm{s}}$ is the sound speed, $\langle a\rangle$ is the averaged minor radius and $\kappa$ is the plasma elongation.

Figure 3 shows the time evolution of $\chi_{i}$ averaged over the whole plasma. Each line represents a different simulation with one of the five values of the total current. The five curves show a similar behaviour, with a transient phase characterized by a series of bursts typical of an avalanche-like behaviour. After this transient phase, a saturated, turbulent quasisteady regime is established. The time scale of the evolution of the heat flux differs from one simulation to another. In order to make physically significant comparisons, we need to 


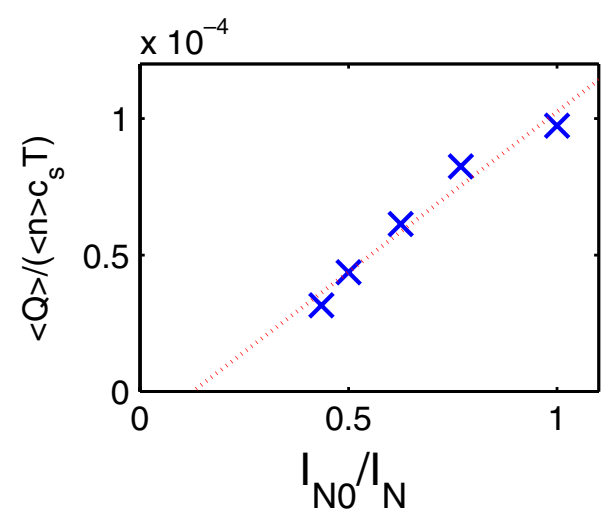

Figure 4. Averaged heat flux in the burst regime, as a function of the inverse total current (results from global nonlinear simulations). The dotted line is a linear fit, $y=a x+b$, with coefficients $a=1.1 \times 10^{-4}, b=-1.5 \times 10^{-5}$.
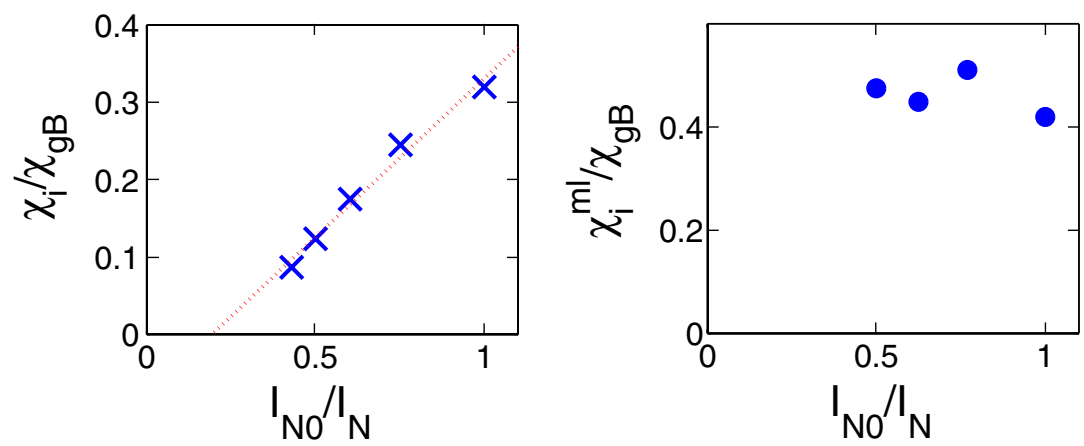

Figure 5. Radial averaged $\chi_{\mathrm{i}} / \chi_{\mathrm{gB}}$ in the burst regime, as a function of the inverse total current. Circles represent the results from linear global calculations, while crosses are results from global nonlinear simulations. The dotted line is a linear fit, $y=a x+b$, with coefficients $a=0.41$, $b=-0.08$.

choose physically equivalent times in the five cases. For this purpose, we choose two time intervals on each curve: one in the burst regime $(a)$ and the other at the beginning of the saturated phase $(b)$. These are the basic points of our analysis and they are plotted in figure 3 . For $\Omega_{\mathrm{ci}} t>9000$ the numerical noise accumulation makes the simulation results unreliable as the energy conservation becomes worse and worse. A new series of simulations, using an optimized loading [15] to reduce noise for long simulations, has been performed for the cases $1 \mathrm{x}$ current and $2 \mathrm{x}$ current (figure 3 , right). They show the establishment of a quasi-steady state (c), for which a clear dependence of the heat flux on plasma current is also observed. We start with the analysis of the value of $\chi_{i}$ averaged over the radial region $(0.3 \leqslant \rho \leqslant 0.8)$ where $\nabla T_{\mathrm{i}}$ and the turbulence have their maximum value. The fluctuations in $\chi_{\mathrm{i}}$ make it difficult to compare its values across the simulations. To partly overcome this problem, the values plotted in figures 4, 5 and 6 represent an average over several periods of these oscillations. In the burst phase, figure 5 shows a linear dependence of the averaged $\chi_{i}$ on the inverse of the total current. The same dependence can be found in the transition phase and in the quasi-steady regime, figure 6 . 


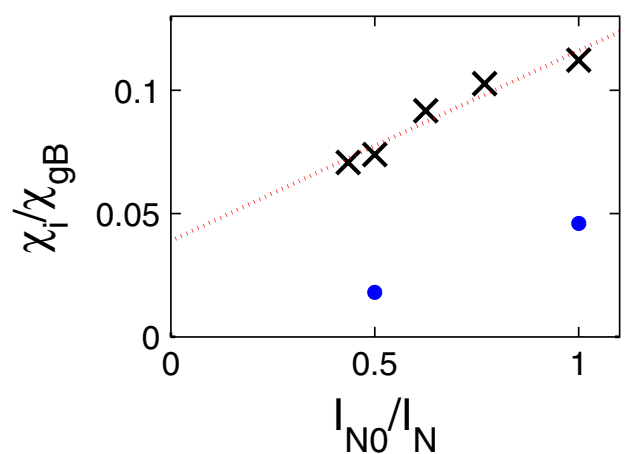

Figure 6. Radial averaged $\chi_{\mathrm{i}} / \chi_{\mathrm{gB}}$ in the transition phase $(x)$ and in the quasi-steady state $(\bullet)$, as a function of the inverse total current. The dotted line is a linear fit, $y=a x+b$, with coefficients $a=0.077, b=0.038$.

Linear simulations have been run on the same equilibrium with the global linear gyrokinetic PIC code GYGLES [25]. The linear value for $\chi_{i}$ is estimated using a mixing length argument. In its simplest form, the mixing length estimate of diffusion coefficients consists of approximating the spatial correlation length and time fluctuation of the turbulent state with the typical perpendicular scale length $\left(k_{\perp}^{-1}\right)$ and the linear growth rate $(\gamma)$ and considering the maximum value of the ratio over the linear spectrum of modes:

$$
\chi_{\mathrm{i}}^{\mathrm{ml}} \simeq \frac{\gamma}{k_{\perp}^{2}}
$$

Practically, for each value of the plasma current a toroidal mode number $(n)$ scan is performed. For each $n$, the most unstable mode is found with its growth rate $\gamma$, and the corresponding $k_{\perp}$ is calculated from the eigenmode structure. Therefore, we have the value of $\gamma / k_{\perp}^{2}$ for each toroidal mode $n$. We, then, define $\chi_{\mathrm{ml}}$ as the largest value of $\gamma / k_{\perp}^{2}$ found over the mode numbers $n$. The found $\chi_{\mathrm{ml}}$ as a function of the total plasma current value is nearly constant (figure 5, right), suggesting that nonlinear phenomena are also affected by the plasma current. Among the possible nonlinear phenomena, interactions of ITG modes with zonal flows are known to play a major role. For the same parameters, nonlinear simulations in which the $n=0$ component was artificially cancelled result in peak values of heat diffusivities too large by a factor $\approx 4$ as compared with the simulations (figure 3 ) in which the $n=0$ was retained. So plasma current appears to have an effect on turbulence also through the interactions with zonal flows. The purpose of the following two sections of the paper is to analyse these effects in more detail.

\section{Zonal flow analysis}

As ITG turbulence evolves from the initial linear phase to a nonlinear stage, zonal flows are generated. In the nonlinear stage, two regimes can be identified. In the first part, a series of bursts in the heat flux can be observed; later, a turbulent quasi-stationary saturated state is set in place. In both the phases there are evidences of a strong correlation between the heat flux and the zonal flows. In this section, we restrict the analysis to the case with $1 \mathrm{x}$ total current, since the features we will describe are common to all the five current cases. A detailed picture of the burst regime is given in figure 7 and the phase of transition between the two regimes has been depicted in figure 8 . We start from the burst regime (between $\Omega_{\mathrm{ci}} t=3460$ and $\Omega_{\mathrm{ci}} t=6860$, figure 7), and we focus on the radial position $\rho=0.5$, 
(a)

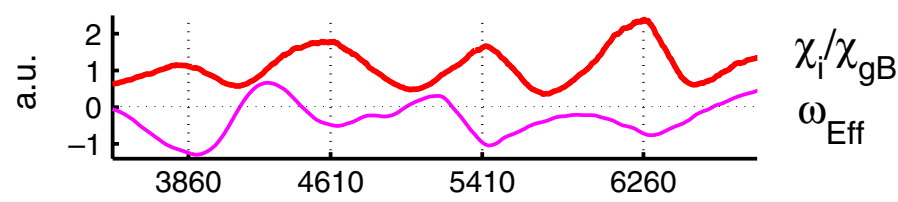

(b)

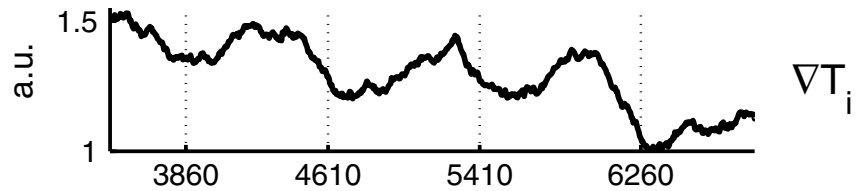

(c)

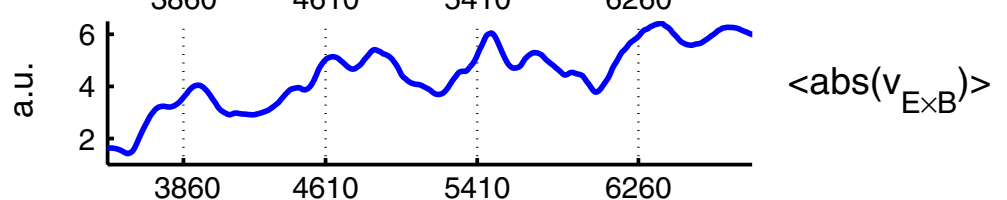

(d)

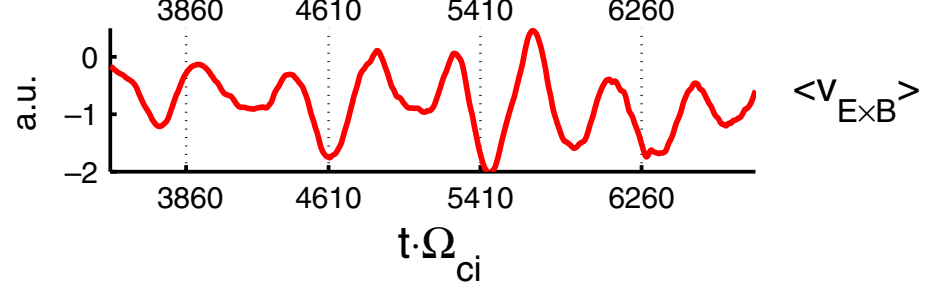

Figure 7. (a) Time evolution of $\chi_{\mathrm{i}}$ and effective shearing rate at radial position $\rho=0.5$, during the burst phase. (b) Temperature gradient at $\rho=0.5$. (c) Radial averaged absolute value of $E \times B$ velocity, showing bursts in the zonal flows. (d) Radial averaged $E \times B$ velocity showing coherent oscillations. The vertical lines indicate the burst positions across the different plots. Vertical scale units are arbitrary.

where the initial temperature gradient has its highest value. From figures $7(a)$ and $(b)$, the maxima of heat conductivity coincide with the phases of decreasing temperature gradient. The following phases of increasing temperature gradient are due to the fact that the local flattening of the temperature is accompanied by local steepening in its neighbourhood. This is characteristic of an avalanche-like regime. The temperature gradient locally peaks until an avalanche is triggered, and, locally, the ITG turbulence grows. The associated heat transport then flattens the temperature gradient and the turbulence is reduced. Then the temperature gradient peaks again and another avalanche is triggered. The sequence continues as long as there is enough free energy to maintain it. After each burst, the temperature gradient does not recover completely and the sequence of bursts stops when the profiles have relaxed to a sufficiently low level. At this point the system has reached a turbulent quasi-steady state. In this picture, the zonal flow oscillations in figure 7(c) are driven by the turbulence oscillations. If this is the mechanism underlying the burst sequence, reducing the initial temperature gradient under a certain critical value should make the bursts disappear. In fact a set of simulations run to check this point shows that a lower gradient reduces the burst amplitude but does not eliminate them. Moreover, in the case where the zonal flows are artificially suppressed (by Fourier filtering the mode $n=0$ in the electrostatic potential), the bursts are suppressed. This suggests a different explanation of the phenomenon. Let us consider the effective shearing rate of the $E \times B$ velocity [8], which is also plotted in figure 7(a). In the burst phase the ITG turbulence grows until the shearing rate exceeds the ambient turbulence decorrelation rate. ITG turbulence and associated transport are then significantly reduced. Now we need a damping mechanism for the zonal flows, in order for the shearing rate to decrease below the decorrelation rate. At this point, ITG turbulence could grow again and start a new burst. But, 


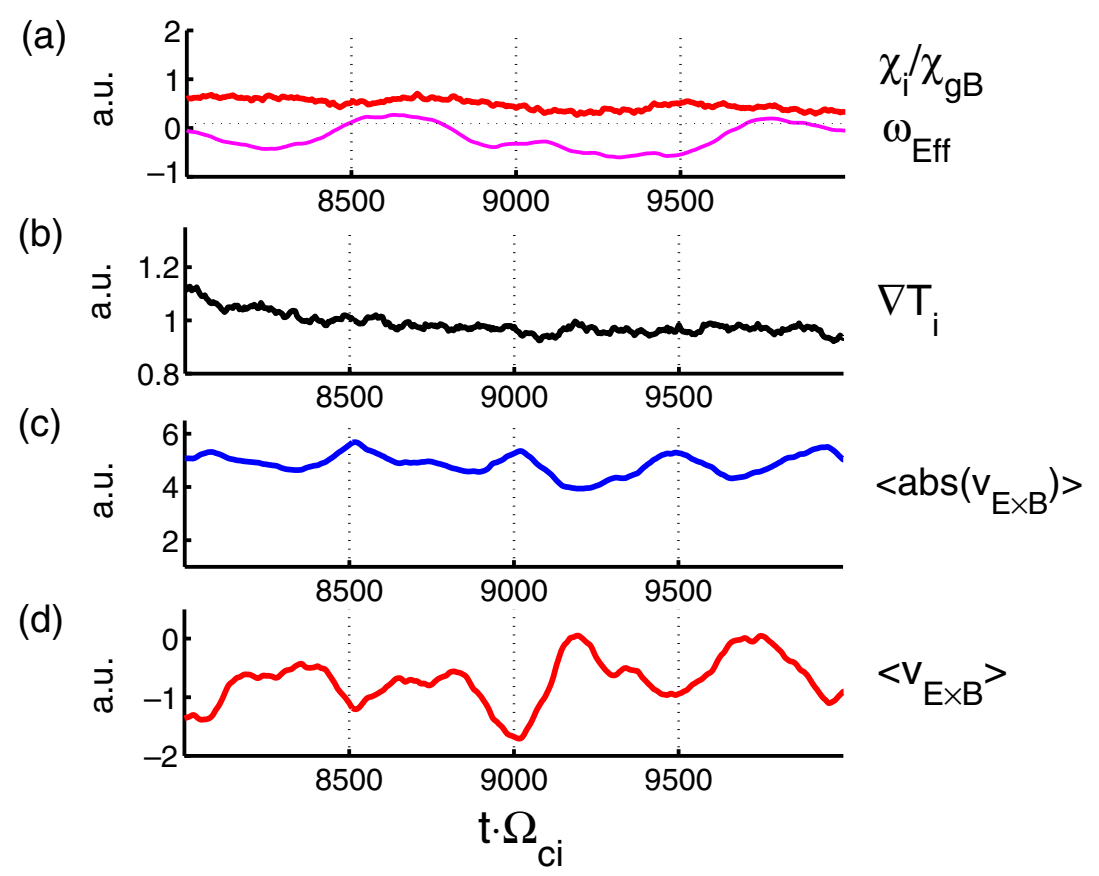

Figure 8. (a) Time evolution of $\chi_{\mathrm{i}}$ and effective shearing rate at radial position $\rho=0.5$, during the transition phase. (b) Temperature gradient at $\rho=0.5$. (c) Without the bursts, the coherent oscillations can now be observed also in the absolute value of the radial averaged $E \times B$ velocity. (d) Radial averaged $E \times B$ velocity. Vertical scale units are arbitrary.

zonal flows are not damped by collisionless processes (Landau damping) [20]. Nevertheless, $E \times B$ velocity oscillations (figure $7(d)$ ), due to coupling to GAMs, can bring the effective shearing rate below the needed level and, together with a peaking in the temperature gradient, can allow for the ITG turbulence to grow again. This burst behaviour is similar to the one found in the presence of collisions [26]. However, here, the zonal flow modulation comes from GAM oscillations instead of a collisional damping.

Bursts observed in zonal flows have a non coherent radial structure. But on top of this, zonal flows have other fluctuations with a coherent radial structure. Both kinds of oscillations can be separated by plotting the time evolution of the radial averages of $E \times B$ velocity and of its absolute value (figure 10). In the first case (bottom line), the radially non-coherent structures cancel out, showing the coherent oscillations which, in this particular case, do not have a zero average value. In the second case (top line) the bursts are instead visible. After a succession of bursts the temperature gradient has relaxed to values close to marginal stability (figure 9 shows the temperature gradient profile evolution). The burst regime is then replaced by a quasi-steady state regime. The collisionless quasi-steady state is characterized by a mean flux and constant entropy production, and it is regarded as an idealization of the real steady state where the high-order moments saturate due to collisional dissipation. A comparison between kinetic simulations in the collisionless limit and in the low-collisionality limit definitely confirms the conventional hypothesis that, for sufficiently low collision frequencies, statistical behaviours of the low order moments such as the transport flux agree with those in the collisionless turbulence $[27,28]$. 


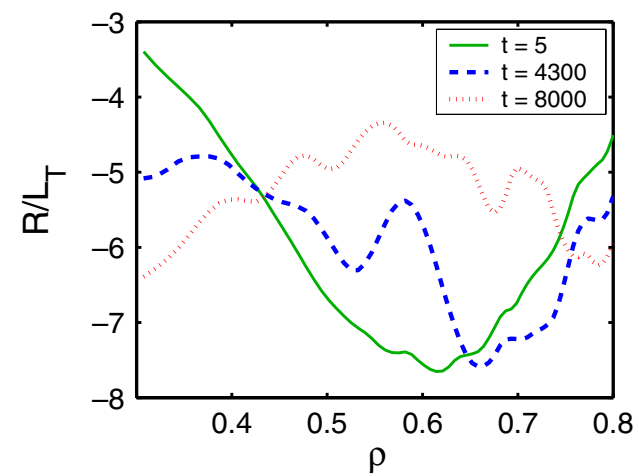

Figure 9. Temperature gradient profile evolution in time. The solid line corresponds to the beginning of the simulation. The dashed line corresponds to the burst phase (the local flattening due to a burst is clearly visible). The dotted line is the relaxed profile at the beginning of the quasi-steady state.

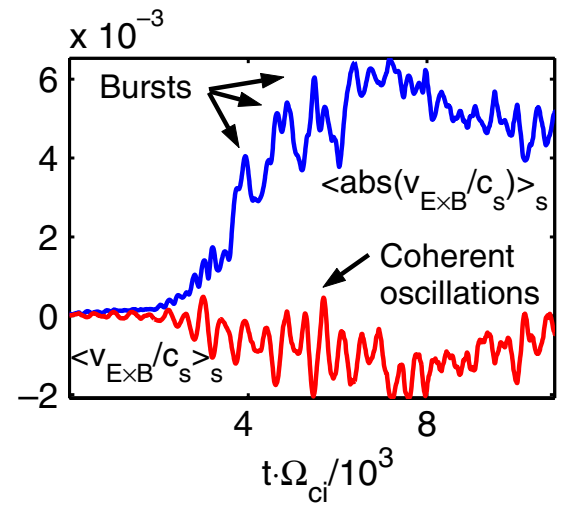

Figure 10. Radial averaged $E \times B$ velocity (lower line) and its absolute value (upper line) as a function of time. Velocities are normalized to the sound speed $c_{\mathrm{s}}$ and time is normalized to $\Omega_{\mathrm{ci}}$.

At the beginning of the quasi-steady phase (figure 8), we observe the coherent fluctuations also in the average $E \times B$ velocity absolute value. In this phase they are no more hidden by the larger bursts. Figures 7 and 8 show how ion diffusivity does not respond directly to the coherent oscillations. Their possible influence on the average transport level will be the topic of the next section.

\section{GAM analysis}

A time spectral analysis has been performed in order to evaluate the frequency of the coherent fluctuations in the averaged $v_{E \times B}$ velocity. The frequency spectrum has been computed over a time interval of $2560 \Omega_{\mathrm{ci}}$, in the late, quasi-steady phase. For each case, a peak in the frequency spectrum has been identified, corresponding to the coherent oscillations. The result, $\omega=$ $0.011 \pm 0.003 \Omega_{\mathrm{ci}}$, is the same in all four simulations and it corresponds to the geodesic acoustic mode frequency. Indeed, an expression for GAM frequency is given in [10], which yields

$$
\omega_{\mathrm{GAM}} \simeq \sqrt{2} \frac{c_{\mathrm{s}}}{R} \simeq 0.011 \Omega_{\mathrm{ci}}
$$




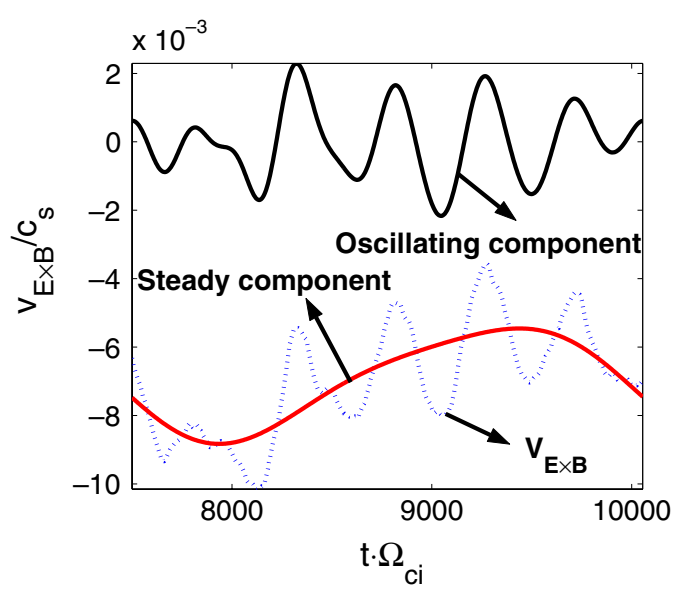

Figure 11. Example of $E \times B$ velocity decomposition at radial position $\rho=0.7$. The full signal is plotted with a dashed line, while the upper and lower solid lines are, respectively, the component oscillating at the GAM frequency and the steady one. Values are normalized to the sound speed $c_{\mathrm{s}}$.
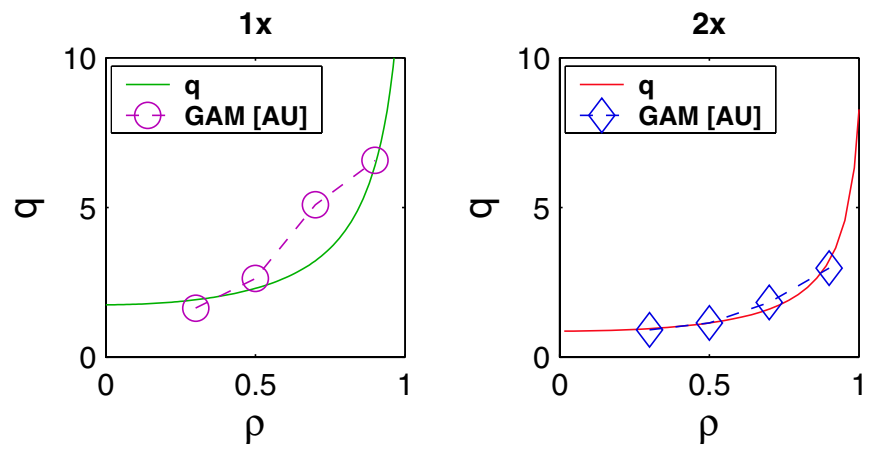

Figure 12. Averaged value of the GAM amplitude, in arbitrary units, as a function of the normalized radial coordinate $\rho$. Diamonds represent results from the $1 \mathrm{x}$ simulation and circles from the $2 \mathrm{x}$ simulation. Values are normalized to the sound speed $c_{\mathrm{s}}$. As a reference, the corresponding safety factor profiles are plotted.

We remark that in the run $1.3 \mathrm{x}$, the frequency found is twice that of the other cases, showing that the plasma can oscillate also at the second GAM harmonic. Equation (6) is the limit for $q \gg 1$ of the more general formula found by Winsor et al [7], where the effects of the parallel dynamics are also included:

$$
\omega_{\mathrm{GAM}}^{2} \simeq 2 \frac{c_{\mathrm{s}}^{2}}{R^{2}}\left(1+\frac{1}{2 q^{2}}\right) .
$$

For the values of $q$ chosen in our simulations, the predicted $\omega_{\mathrm{GAM}}$ depends very weakly on the value of the rotational transform and correspondingly on the total current.

We now turn to the analysis of the effects of plasma current on the GAM amplitude. First, we separate the steady part from the oscillatory part of the zonal flows. We apply a Fourier filter on the $v_{E \times B}$ velocity signal, in order to isolate the GAM oscillations from the slowly varying component. The results are plotted in figure 11, where the steady and oscillating components are plotted together with the original total signal. The GAM amplitude is then evaluated taking the average of the peak to bottom difference over a series of oscillations. In figure 12 we plot 


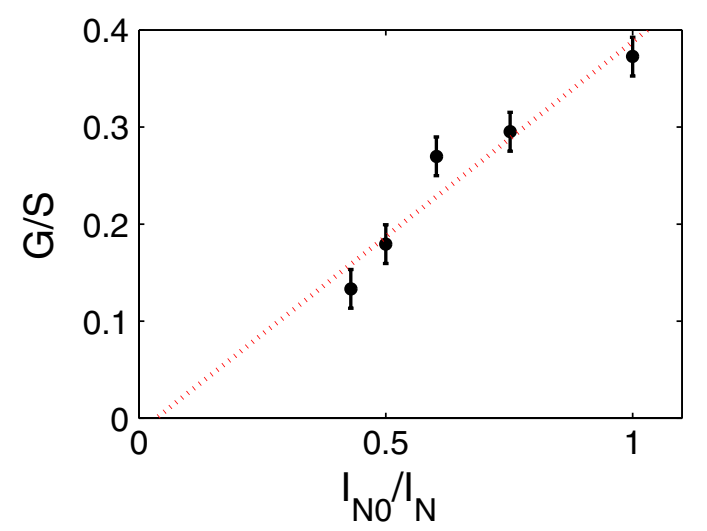

Figure 13. Ratio of the averaged GAM amplitude (G) to the steady zonal flow component (S) in the burst regime as a function of the inverse total current. The dotted line is a linear fit, $y=a x+b$, with coefficients $a=0.40, b=0.014$.

the averaged value of GAM amplitude as a function of the normalized radial coordinate $\rho$. The radial profile of the GAM amplitude is clearly correlated with the value of $q$. The GAM amplitude increases with increasing $q$. In [10], global Landau-fluid simulations are presented showing that the zonal flow behaviour changes as a function of the safety factor $q$. According to the equations derived in the same reference, $(m, n)=(1,0)$ pressure perturbations drive comes from three terms. One is a nonlinear coupling with turbulence, the second is the coupling with zonal flows through geodesic curvature (GAM term) and the third is a coupling with sound waves (sound wave term). The latter term is opposite to the GAM one and inversely proportional to $q$, so at low $q$ it becomes large and can balance the GAM term, preventing the excitation of the $(1,0)$ pressure mode by the zonal flow. At high $q$, where the sound wave term is negligible, zonal flows can excite $(1,0)$ pressure perturbations. As a result, in the low $q$ region the stationary zonal flows are dominant, while in high $q$ region the zonal flows are mainly oscillatory due to the coupling with the $(1,0)$ pressure perturbations. This result is reproduced in our gyrokinetic simulations as shown in figure 12 . However, for the high values of $q$ chosen in our simulations (up to $q=16.55$ ), the competition between parallel dynamics and curvature may not be a strong enough argument to explain, alone, this result. A support to this statement is that the $q^{2}$ dependence in equation (7) is very weak in the present set of simulations (even if observed during the Rosenbluth-Hinton test for the ORB5 code, for lower $q$ values). A kinetic effect can contribute substantially to the observed dependence of the GAM amplitude: the GAM oscillations $(n=0, m= \pm 1)$ are linearly damped through a collisionless Landau damping mechanism. For large values of $q(q \geqslant 2)$, the collisionless damping becomes exponentially small [29]:

$$
\frac{\gamma_{\mathrm{GAM}}}{\omega_{\mathrm{GAM}}} \simeq \exp \left(-q^{2}-\frac{1}{2}\right)
$$

For lower $q$ values, the GAM are strongly damped, and only the stationary $(n=0, m=0)$ zonal flow component survives.

Now, we can ask the question if the link between GAM amplitude and safety factor could be extended to the total plasma current value. From the five simulations with different total plasma current values, we plotted the radial averaged ratio of the GAM amplitude $(\mathrm{G})$ to the steady zonal flow component $(\mathrm{S})$ as a function of inverse total current. Figures 13 and 14 refer, respectively, to burst and transition plus stationary phases. The fact that the $q$ profile scales 


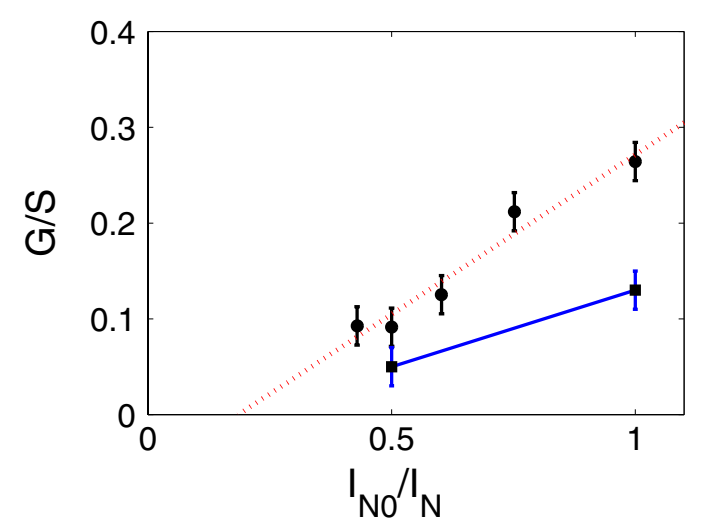

Figure 14. Ratio of the averaged GAM amplitude $(\mathrm{G})$ to the steady zonal flow component $(\mathrm{S})$ as a function of the inverse total current. Dots represent data from the transition phase and squares from the quasi-steady regime. The dotted line is a linear fit, $y=a x+b$, with coefficients $a=0.33$, $b=-0.061$.

with the inverse total current and that GAM amplitude is proportional to the $q$ value, reflects on the linear dependence of G/S on the inverse total current. This brings us back to the scaling of the average ion heat transport with the total plasma current $I_{\mathrm{p}}$. In section 4 , it has been shown that it is inversely proportional to $I_{\mathrm{p}}$. This nonlinear result can now be understood in terms of interactions between zonal flows oscillation and ITG turbulence. In ref. [8] it has been shown that, while the stationary zonal flows suppress the turbulence efficiently, fluctuations make the suppression of the turbulence by the zonal flows less effective. Increasing $I_{\mathrm{p}}$ reduces the GAM amplitude, and the zonal flow behaviour moves from oscillatory to stationary resulting in a lower radial ion heat transport. This effect is global in our simulations, and it can be observed in both the burst phase and in the quasi-steady regimes.

\section{Conclusions}

A set of nonlinear electrostatic ITG turbulence simulations has been run with the global gyrokinetic PIC code ORB5. Two regimes can be distinguished in the nonlinear phase of the turbulence development. The first is characterized by a series of bursts in the averaged radial heat flux, the second is characterized by a turbulent quasi-steady state. A burst starts in correspondence with a minimum of the $E \times B$ shearing rate. The turbulence level locally grows and it drives the zonal flows until the shearing rate exceeds the linear growth rate. ITG turbulence and the associated heat transport are then substantially reduced. After having been locally flattened, the temperature profile can peak again because of other bursts in the neighbouring radial position. The increased temperature gradient, and the lower shearing rate due to a minimum in the GAM oscillations, allows the turbulence to grow again and a new burst is started. The averaged value of the ion conductivity and the volume-averaged ion radial heat flux are inversely proportional to the total plasma current. The same dependence on total plasma current is found for the amplitude of zonal flows' oscillations at the GAM frequency. When the relaxation of the temperature profile leads the ITG modes close to the marginal stability, a turbulent quasi-steady state sets in. The ion diffusivity and heat flux assume a steady level while zonal flows oscillate at the GAM frequency. The average ion diffusivity level and the GAM amplitude are inversely proportional to the total current. This relation found both in the 
burst phase and in the stationary state suggests a common underlying transport mechanism, while the transition between the two phases is determined by the proximity of the temperature gradient to its critical value. This transition can be observed because, in the model and in its numerical implementation, the temperature profile is not fixed, but it is determined by the dynamical evolution of the system. On the other hand, without a heat source, we are not able to study directly the effect of a forced turbulence. Nevertheless, the study presented in this paper allows us to infer some qualitative proprieties also for the case of a forced turbulence. While the temperature gradient relaxes from the initial value to a value close to the critical one, we observe, at first, a phase of strong turbulence, dominated by avalanches and linear drive effects, and, in a second time, a phase where the turbulence is close to the stability threshold, dominated by the effects of the zonal flows. The presence of a strong heating source would act as a restoring force which would bring back the temperature gradient to its value after each avalanche, thus maintaining a burst regime. A less powerful source would keep the temperature gradient close to marginal stability. Our simulations suggest that in both the cases the total current plays an important role in regulating the zonal flow behaviour and the ion heat transport.

The set of simulations gives a coherent picture in which the ion turbulent heat transport is linked to the amplitude of the zonal flow GAM oscillations through the total plasma current value (and correspondingly through the safety factor profile). The safety factor determines the behaviour of the zonal flows between stationary and oscillatory. A low total plasma current allows for oscillatory zonal flows which are less effective in shearing the turbulence leading to higher transport while a high value in the current leads to stationary zonal flows and a reduced transport. Plasma current has an effect not only on GAM oscillations of zonal flows but also on important quantities such as the orbit widths and the linear stability spectrum. The obtained dependence of the anomalous radial heat transport on the plasma current should be seen as resulting from a combination of these effects, all of which are included in our simulations.

The relationship found between zonal flow oscillatory behaviour and the safety factor profile may play an important role in the formation of internal transport barriers when a reversed shear profile is considered. The plasma shape can also play a role in the interactions between zonal flows and GAM (while toroidicity couples $m=0$ with $m= \pm 1$, plasma elongation couples $m=0$ to $m= \pm 2$ ). Another important example is the study of the scaling of transport with the device size ( $\rho^{*}$ scaling), extensively discussed by several authors [30-32], and that has already given important even if debatable results. All these interesting questions deserve a detailed analysis and will be addressed in future work.

\section{Acknowledgments}

This work has been partly supported by the Swiss National Science Foundation. The simulations have been run on the Pleiades cluster and the IBM Blue Gene parallel machine at EPFL.

\section{References}

[1] Garbet X 2001 Plasma Phys. Control. Fusion 43 A251

[2] Romanelli F 1989 Phys. Fluids B 11018

[3] Hasegawa A and Wakatani M 1987 Phys. Rev. Lett. 591581

[4] Burrell K 1999 Phys. Plasmas 64418

[5] Lin Z, Hahm T, Lee W, Tang W and White R 1998 Science 2811835 
[6] Diamond P H, Itoh S I, Itoh K and Hahm T S 2005 Plasma Phys. Control. Fusion 47 R35

[7] Winsor N, Johnson L J and Dawson M J 1968 Phys. Fluids 112448

[8] Hahm T S et al 1999 Phys. Plasmas 6922

[9] Scott B 2003 Phys. Lett. A 32053

[10] Miyato N and Kishimoto Y 2004 Phys. Plasmas 115557

[11] Tran T M et al 1999 Theory of fusion plasmas Int. Workshop (Bologna: Editrice Compositori, SIF) p 45

[12] Hahm T 1988 Phys. Fluids 312670

[13] Bottino A et al 2004 Recent advances in nonlinear gyrokinetic PIC simulations in tokamak geometry in theory of fusion plasmas Int. Workshop (Bologna: Editrice Compositori, Societa' italiana di Fisica) p 75

[14] Angelino P et al 2004 Geometrical coupling of zonal flows on electrostatic microinstabilities in theory of fusion plasmas Int. Workshop (Bologna: Editrice Compositori, Societa' italiana di Fisica) p 329

[15] Hatzky R et al 2002 Phys. Plasmas 9898

[16] Allfrey S and Hatzky R 2003 Comput. Phys. Commun. 15498

[17] Idomura Y, Tokuda S and Kishimoto Y 2003 Nucl. Fusion 43234

[18] Lütjens H, Bondeson A and Sauter O 1996 Comput. Phys. Commun. 97219

[19] Jolliet S, Angelino P, Bottino A, Idomura Y and Villard L 2004 A linear gyrokinetic model in magnetic coordinates in theory of fusion plasmas Int. Workshop (Bologna: Editrice Compositori, Societa' italiana di Fisica) p 329

[20] Rosenbluth M N and Hinton F L 1998 Phys. Rev. Lett. 80724

[21] Bottino A, Sauter O, Camenen Y and Fable E 2005 Plasma Phys. Control. Fusion 48215

[22] Bottino A 2004 Modelling of global electrostatic microinstabilities in tokamaks: effects of exb flow and magnetic shear PhD Thesis No. 2938, Ecole Polytechnique Fédérale de Lausanne

[23] Wesson J 1997 Tokamaks 2nd edn (Oxford: Clarendon)

[24] Waltz R E, DeBoo J C and Rosenbluth M N 1990 Phys. Rev. Lett. 652390

[25] Fivaz M et al 1998 Comput. Phys. Commun. 11127

[26] Lin Z, Hahm T S, Lee W W, Tang W M and Diamond P H 1999 Phys. Phys. Rev. Lett. 833645

[27] Watanabe T-H and Sugama H 2002 Phys. Plasmas 93659

[28] Watanabe T-H and Sugama H 2004 Phys. Plasmas 111476

[29] Lebedev V B, Yushmanov P N, Diamond P H, Novakovskii S V and Smolyakov A I 1996 Phys. Plasmas 33023

[30] Lin Z, Ethier S, Hahm T and Tang W 2002 Phys. Rev. Lett. 88195004

[31] Dimits A M, Williams T J, Byers J A and Cohen B I 1996 Phys. Rev. Lett. 7771

[32] Candy J and Waltz R 2003 Phys. Rev. Lett. 91045001 